\title{
Collection of Ice Particles from Aircraft Using Decelerators ${ }^{1}$
}

\author{
Peter V. Hobbs, ${ }^{2}$ Robert J. Farber ${ }^{2}$ and Robert G. Joppa ${ }^{3}$ \\ Universily of Washington, Seattle 98195
}

(Manuscript received 4 October 1972, in revised form 11 December 1972)

\begin{abstract}
The design, evaluation and field testing of two devices (called decelerators) for reducing the velocities with respect to an aircraft of airborne cloud and precipitation particles are described. With the aid of these decelerators delicate ice crystals in excess of $1 \mathrm{~mm}$ in size can be collected without breakage from aircraft flying at $50 \mathrm{~m} \mathrm{sec}^{-1}$, and somewhat smaller ice crystals can be collected intact at aircraft speeds up to $100 \mathrm{~m} \mathrm{sec}^{-1}$.
\end{abstract}

\section{Introduction}

A widely used, and extremely useful, technique for sampling cloud and precipitation particles from aircraft is to allow the particles to impact on a moving Mylar film where they are encapsulated in a Formvar plastic solution and subsequently replicated (e.g., MacCready and Todd, 1964). However, a serious limitation of this technique is that even at fairly low aircraft speeds many ice crystals larger than about $100 \mu \mathrm{m}$ in maximum dimension shatter when they impact on the Formvarcoated film. This not only makes detailed studies of the crystals impossible but adds an additional complication to the already difficult problem of estimating the concentrations of ice particles in the air from the number of particles collected on the film. One method of reducing fragmentation is to lower the velocities of the particles in the air with respect to the aircraft by reducing the airflow past the film. Such a device is referred to as a decelerator.

Yamashita (1969) described a decelerator in which the flow of air into a tube containing the Formvarcoated film was restricted so that a reverse airflow was set up which reduced the relative velocity of the particles with respect to the aircraft. The amount of deceleration and the maximum sizes of ice crystals collected intact were not given, although the photographs accompanying the paper show some unbroken plate-like crystals up to about $250 \mu \mathrm{m}$ in maximum dimension. However, it would appear from the brief description which Yamashita gives of this device, that the distance over which the particles would have experienced a decelerating force was rather small.

Mossop et al. (1967) designed and tested two decelerators. The first (A) consisted of a sampling tube with an

\footnotetext{
${ }^{1}$ Contribution No. 263, Department of Atmospheric Scicnces, University of Washington.

2 Department of Atmospheric Sciences.

Department of Aeronautics and Astronautics.
}

intake diameter of $2.5 \mathrm{~cm}$ which flared out at a semiangle of $2^{\circ}$ to a diameter of $8.3 \mathrm{~cm}$. The entrance tube was parallel to the axis of the aircraft for a distance of $2 \mathrm{~m}$ and then it bent at right angles and passed into the aircraft. The air speed near the exit of the tube was $30 \%$ of the aircraft speed. The second decelerator (B) was a straight tube with an intake diameter of 4.1 cm which flared out at a semi-angle of $3^{\circ}$ to a final diameter of $9.9 \mathrm{~cm}$. The length of the tube was $3.7 \mathrm{~m}$ and the particles were collected at a distance of $27 \mathrm{~cm}$ from the end of the tube. The velocity of the air with respect to the aircraft was reduced by a factor of 3 in this device. Finally, Mossop and Ono (1969) designed a decelerator $(C)$ which consisted of two coaxial tubes of internal diameter 14.8 and $7.4 \mathrm{~cm}$, with the annular space between the tubes closed at the front end. With the aid of a fan connected to the outer tube, air could be drawn out of the inner tube through holes in its wall so that any desired fraction of the air entering the front end of the central tube could be removed, thereby decelerating the axial velocity of the air. This decelerator reduced the velocity of impact of the particles on the collector to about one-third the aircraft speed.

Of the three decelerators referred to above it appears that the best results were obtained with decelerator B. The main fault with decelerator $A$ was probably the sharp bend in the tube where ice particles may have collided with the walls of the tube and fragmented. The relative failure of decelerator $\mathrm{C}$ was ascribed to its narrowness in relation to its length. However, even with decelerator B, Mossop et al. state that crystal shattering was still apparent and was probably due to turbulence in the diffuser section and to the ice particles striking the walls.

In this paper we describe two decelerators in which the relative velocity of the air is reduced to one-fifth of the aircraft speed. With the aid of these decelerators 
we have collected from aircraft delicate ice crystals in excess of $1 \mathrm{~mm}$ in size without crystal fragmenting.

\section{Design of the decelerators}

In designing the decelerators the following requirements were considered: (i) the velocity reduction ratio at the sampling point must be predictable, (ii) turbulence in the air should be kept to a minimum in order to avoid violent crystal motions and possible break-up, and (iii) deceleration should be fairly slow. These requirements taken together imply that the duct contours of the decelerator should be smooth so that nearly potential flow will exist, and that pressure gradients must not be severe. Under these conditions a one-dimensional flow will exist in the duct.

A discussion of the design properly begins at the exit or tailing edge, and proceeds forward to the entrance. The Kutta condition at the trailing edge insures that the exit velocity from the duct will be equal to the free stream velocity (e.g., Lamb, 1945). Conservation of mass then requires that the ratio of velocity at the sampling point to that in the free stream be just the inverse of the area ratio of those two points. In order to insure this condition, the external contour of the rear or converging part of the duct must converge slowly enough to prevent separation on the outside surfaces. Experience suggests that the length of this section should be two to four times the maximum diameter and that its contours should be smooth.

A constant area section is provided upstream of the sample point. In this region the air speed will be slow, and will provide sufficient time for the particles to be decelerated and approach the speed of the air.

The stream tube which enters the duct must originally by the same size as the exit stream and become larger as the flow decelerates. This deceleration may be controlled to some desired rate by contouring the entrance cone, or it may be allowed to occur in an uncontrolled

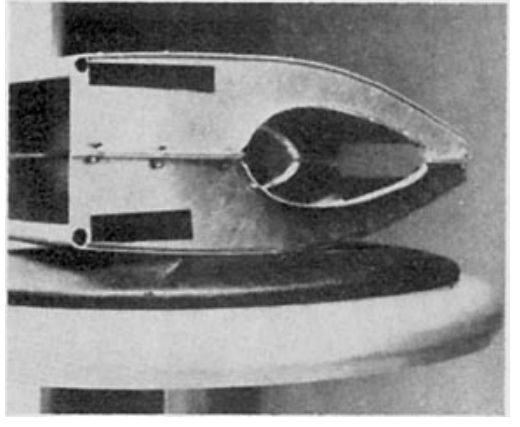

FIG. 1. Decelerator 1. Air enters from left-hand side. Particle sampler is not shown.

manner before entering the decelerator. The choice is made by the way in which the area increases from the entrance to the constant area section. If the entrance cone is too long, turbulence from the wall boundary layer may reach the center line of the duct. If it is too short and the divergence angle is too large, the flow will separate from the walls and turbulence will be generated everywhere in the duct. The leading edges or lips of the duct must be rounded to allow for smooth entry of the air.

Two decelerators were designed using these guidelines. A photograph of the first decelerator (which we will call decelerator 1) is shown in Fig. 1 and a dimensional sketch is shown in Fig. 2. The duct is rectangular and of constant width of $12.7 \mathrm{~cm}$. It was constructed of $1-\mathrm{mm}$ aluminum alloy sheet. The area ratio of the sample point to the exit point is 5 , so the velocity of the air at the sampling point should be one-fifth of the free-stream velocity. No entrance cone is provided beyond the rounding of the leading edge lips, and so the air deceleration is uncontrolled and occurs upstream of entry to the duct. Flow velocities along the center line was measured in a wind tunnel and are presented in Fig. 3. It will be seen that the air deceleration occurs

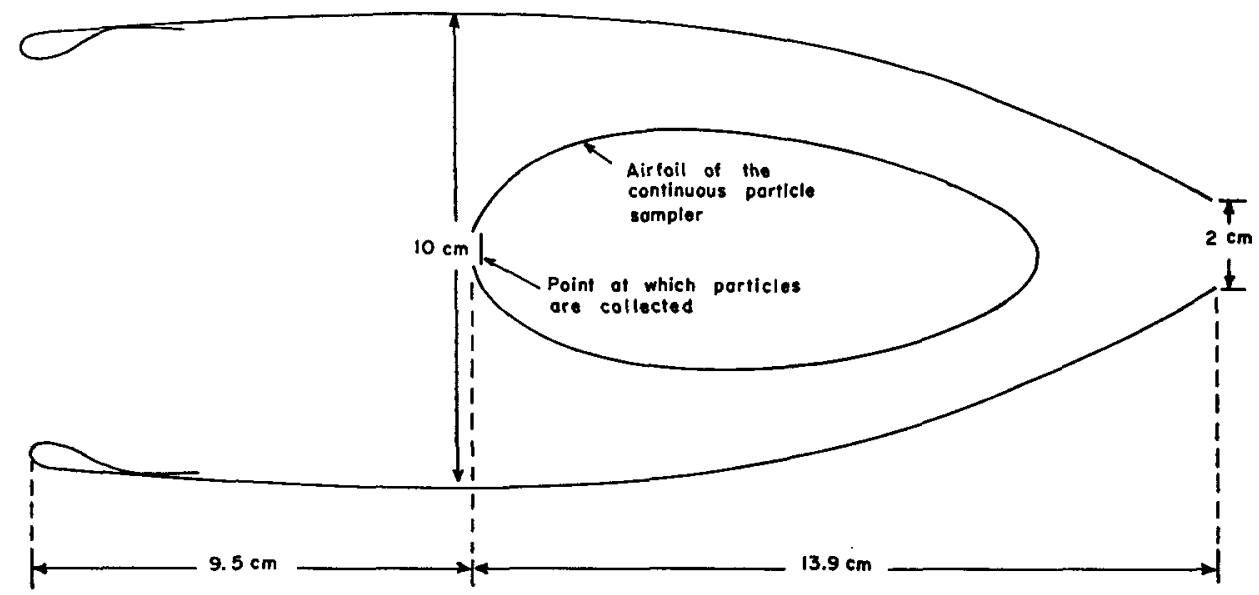

FIG. 2. Cross section along the longer central axis of decelerator 1. The cross section normal to the one shown in this diagram is rectangular with a constant width of $12.7 \mathrm{~cm}$. 


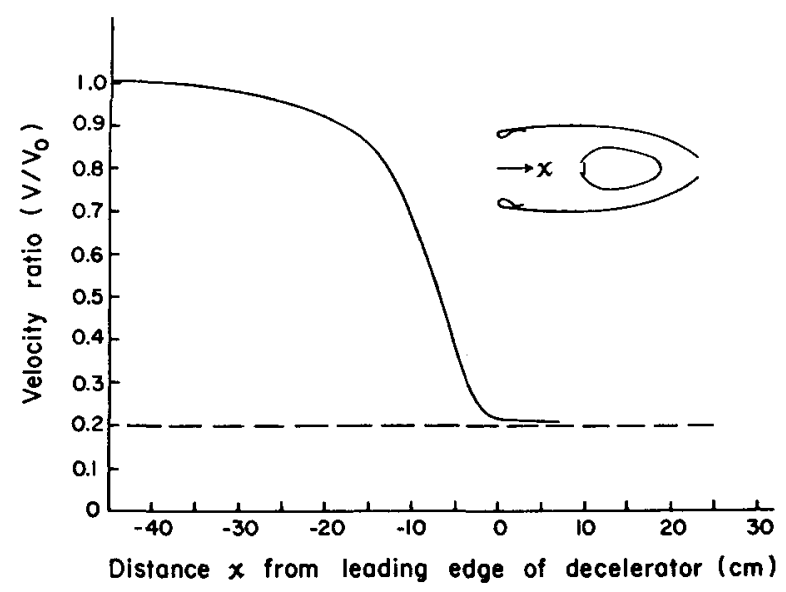

FIG. 3. Velocity of air along center line of decelerator 1 .

almost entirely before entry into the duct, and that the predicted velocity is achieved.

The second decelerator (decelerator 2) is shown in Figs. 4 and 5. This duct is of circular cross section. It was constructed of aluminum tubing of $1.6 \mathrm{~mm}$ thickness, and the entrance and exit cones are of spun aluminum to the given contours. The area ratio is again 5 , giving a velocity ratio of one-fifth. A constant velocity section of two diameters length is provided for deceleration of particles. The air deceleration is controlled over a longer length than in decelerator 1 by an entrance cone which has an area ratio of $2: 2$. Thus, part of the deceleration occurs outside the duct, and the remainder inside.

Wind tunnel measurements were taken along the center line of the duct and are shown in Fig. 6. Note that the air starts to decelerate more than one diameter ahead of the inlet, and that the speed is reduced by about $5^{-\frac{1}{3}}$ before entry and again by about $5^{-\frac{1}{3}}$ after entry.

The length of the entry and of the constant area sections could be longer if desired but they are limited by the risk of turbulence. A critical Reynold's number of about 2000 is exceeded at normal aircraft speeds, but fully developed turbulent flow only occurs at distences in excess of about 25 diameters downstream from the entry to a pipe (Schlicting, 1955). This

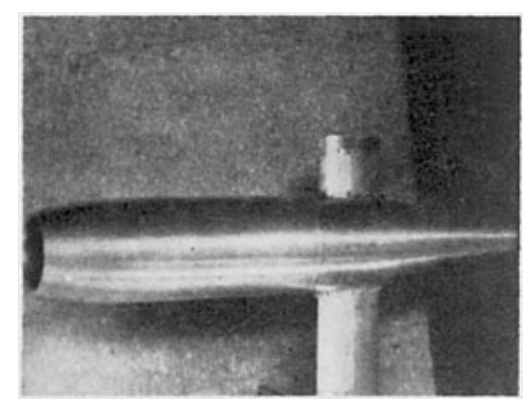

Fig. 4. Decelerator 2 mounted on the particle sampler. Air enters from left-hand side. estimate may be optimistic where the entry is an expansion with its adverse pressure gradient. The pressure gradient is kept small by restricting the effective cone angle to an amount which has proven satisfactory in preventing separated flow in wind tunnels (Bradshaw and Pankhurst, 1964).

When flying in supercooled clouds, ice can collect on the leading edge of a decelerator and disturb the airflow. This may be prevented by heating the rolled leading edges with internal electric heaters.

The collection efficiency of the decelerators for various types and sizes of ice crystals has not been determined; therefore, at the present time, it is not possible to deduce accurate values for the concentrations of ice particles in the air when the decelerators are used.

\section{Retardation of ice crystals}

Although the air entering a decelerator is slowed down relative to the aircraft, ice crystals in the air will be decelerated at a slower rate due to their inertia. In this section we estimate the distances which platelike and column-like crystals of a given size must travel in the decelerators for a given speed reduction.

The air is slowed gradually over some distance, but for simplicity in calculating the response, it will be assumed that the air decelerates in a step-wise manner at a certain point. The calculation is best done from the point of view of moving particles striking stationary air, so a coordinate system will be used which is fixed with respect to the retarded air in the decelerator, and moves at a speed $V_{1}$ with respect to the decelerator itself. Then we may write

$$
-C_{D} \frac{S \rho v^{2}}{2}=m \frac{d^{2} \xi}{d t^{2}}
$$

where $C_{D}$ is the drag coefficient, $S$ the area of the crystal to which the drag coefficient refers, $\rho$ the air density, $v$ the velocity of crystal with respect to the air, $m$ the crystal mass, and $\xi$ the distance the crystal moves through the air. We may substitute

$$
\alpha=\frac{C_{D} \rho S}{2 m}
$$

and note that $d \xi / d t=v$ and eliminate time, writing:

$$
-\alpha d \xi=\frac{d v}{v} .
$$

This is easily integrated between the initial values of $\xi=0, v=v_{i}$ and the general values $\xi$ and $v$, to give

$$
\frac{v}{v_{i}}=\exp (-\alpha \xi) .
$$




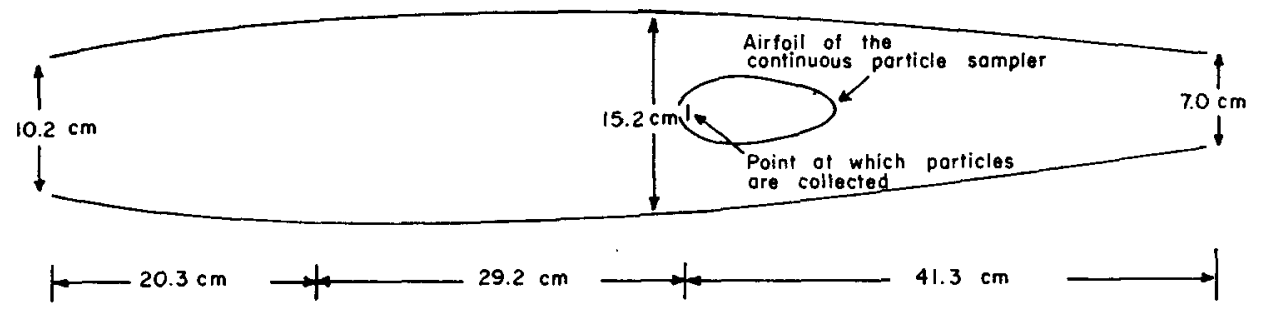

FIG. 5. Cross section along the longer central axis of decelerator 2. The cross section normal to the one shown in this diagram is circular.

Eq. (4) may be interpreted to show a characteristic retardation distance $\xi=1 / \alpha$, at which the velocity ratio $v / v_{i}=\exp (-1)$. However, these velocities are measured with respect to the air which is itself moving at some velocity $V_{1}$ with respect to the decelerator, and we would like to find the velocities of the particle and the distance the particle moves with respect to coordinates fixed in the decelerator.

The distance the particle moves is $\xi$ plus the distance the coordinate system has moved, which is $V_{1} T$. Thus

From (1) and (2)

$$
x=V_{1} T+\xi \text {. }
$$

which on integrating yields

$$
-\alpha v^{2}=\frac{d v}{d t}
$$

$$
T=\frac{1}{\alpha v_{i}}\left(\frac{v_{i}}{v}-1\right)
$$

The total distance moved is then found by using (4) and (6) in (5) to give

$$
x=\frac{V_{1}}{\alpha v_{i}}\left(\frac{v_{i}}{v}-1\right)+\frac{1}{\alpha} \ln \frac{v_{i}}{v} .
$$

Since $v$ and $v_{i}$ are measured in the moving coordinate system, they should be converted to velocities $V$ in the fixed coordinate system, namely

$$
\left.\begin{array}{l}
V=V_{1}+v \\
V_{0}=V_{1}+v_{i}
\end{array}\right\},
$$

where $V_{0}$ is the (remote) velocity of the undisturbed air, $V_{1}$ the internal velocity of retarded air in the decelerator, and $V$ the particle velocity at a distance $x$ from the step-velocity decrease. Then

$$
x=\frac{V_{1}}{\alpha\left(V_{0}-V_{1}\right)}\left(\frac{V_{0}-V_{1}}{V-V_{1}}-1\right)+\frac{1}{\alpha} \ln \left(\frac{V_{0}-V_{1}}{V-V_{1}}\right) .
$$

If we let the decelerator velocity ratio be $R=V_{1} / V_{0}$ and divide (8) by $V_{0}$, we have

$$
x=\frac{1}{\alpha}\left\{\left(\frac{R}{1-R}\right)\left[\frac{1-R}{\left(V / V_{0}\right)-R}-1\right]+\ln \left[\frac{1-R}{\left(V / V_{0}\right)-R}\right]\right\} \text {. }
$$

This characteristic deceleration curve is shown in Fig. 7 . An examination of Fig. 7 shows that the deceleration is extremely rapid, and that for values of $x \alpha \gtrsim 2$, the velocity reduction has been largely completed. We may now examine the uncertainties introduced by the assumption of a step function for the velocity profile. This assumption requires the user to estimate a point on the actual profile at which the particle is assumed to encounter the fully decelerated air. An error in the

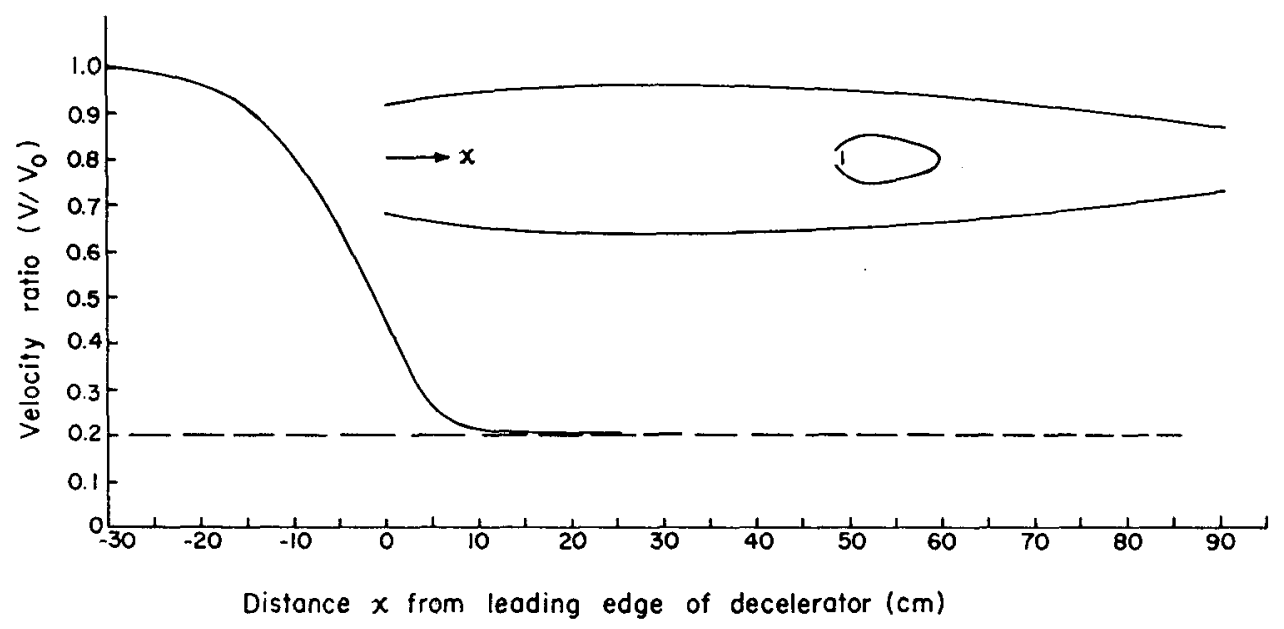

Fic. 6. Velocity of air along center line of decelerator 2. 


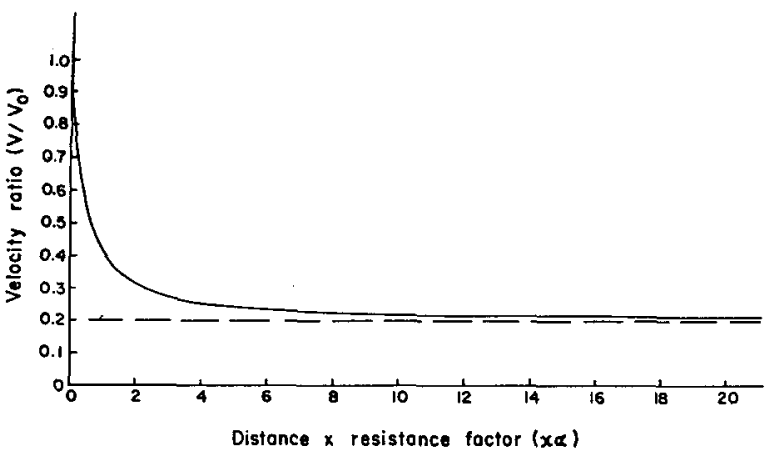

FIG. 7. Deceleration of a particle encountering a sudden reduction of $20 \%$ in air velocity as calculated from Eq. (9).

estimation of this point changes the value of the distance $x$ from that point to the sample collection point. For values of $x_{\alpha}>2$, the slope of the curve in Fig. 7 is seen to be quite small, so that errors in selection of the proper starting point will have small effect on the final value of velocity of the particle.

We now consider the characteristics of the ice crystals. In the case of the hexagonal plates

$$
\frac{S}{m}=\frac{1}{w \rho_{p}}
$$

where $w$ is the thickness of the plate and $\rho_{p}$ its density (which we will take as the density of ice, namely, 0.9 $\left.\mathrm{gm} \mathrm{cm}^{-3}\right)$. The plate may have random orientation at first, but if we assume that it is stable only when its plane is oriented normal to the flow, and that it assumes this position quickly, then the drag coefficient of a disk is appropriate which may be taken as 1.12 (Goldstein, 1938). Taking the density of air at $-10 \mathrm{C}$ and $800 \mathrm{mb}$ $\left(0.0011 \mathrm{gm} \mathrm{cm}^{-3}\right)$, the coefficient $\alpha$ becomes from (2)

$$
\alpha=\frac{0.000685}{w} \text {. }
$$

For a hexagonal plate $1 \mathrm{~mm}$ in diameter, $w=40 \mu \mathrm{m}$ (Auer and Veal, 1970); therefore, $\alpha=0.17$.

From (9), or Fig. 7, it may be seen that the particle is decelerated to about $42 \%$ of the free-stream speed at $6 \mathrm{~cm}$, and to $30 \%$ at about $12 \mathrm{~cm}$ from the point

TABLE 1. Deceleration of plates, columns and stellar crystals with maximum dimension of $1 \mathrm{~mm}$ in decelerators 1 and 2 . See text for definition of symbols.

\begin{tabular}{cclcll}
\hline \hline & & & & & \\
Decelerator & $(\mathrm{cm})$ & Crystal & \multicolumn{1}{c}{$\alpha$} & \multicolumn{1}{c}{$x \alpha$} & $V / V_{0}$ \\
\hline 1 & 17 & Plate & 0.17 & 2.9 & 0.275 \\
1 & 17 & Column & 0.05 & 0.85 & 0.45 \\
1 & 17 & Stellar & $0.15+$ & $2.5+$ & $0.28-$ \\
2 & 53 & Plate & 0.17 & 9.0 & 0.22 \\
2 & 53 & Column & 0.05 & 2.6 & 0.29 \\
2 & 53 & Stellar & $0.15+$ & $8.0+$ & $0.23-$ \\
\hline
\end{tabular}

at which the velocity of the air is assumed to change abruptly.

In the case of a columnar ice crystal of hexagonal cross section, the most favorable orientation is with its length normal to the flow, although it is by no means certain that a crystal will be stable in this attitude. In this position the area $S=l d$ and the mass is

$$
m=2.60\left(\frac{d}{2}\right)^{2} l_{\rho_{c}}
$$

where, $l$ is the column length, $d$ the column diameter, and $\rho_{c}$ the density of the column. Therefore,

$$
\begin{gathered}
\frac{S}{m}=\frac{1.53}{d \rho_{c}} \\
\alpha=0.765 \frac{C_{D} \rho}{d \rho_{c}} .
\end{gathered}
$$

The density of columnar ice crystals is variable since some are solid and others partly hollow. However, for these calculations we will assume $\rho_{c}=0.9 \mathrm{gm} \mathrm{cm}^{-3}$. A columnar ice crystal $1 \mathrm{~mm}$ in length has a diameter of $200 \mu \mathrm{m}$ (Auer and Veal, 1970), and for velocities with respect to the air of from 80 to $30 \mathrm{~m} \mathrm{sec}^{-1}$, the Reynolds numbers are in the range 1100 to 400 . Since the corresponding values of the drag coefficient $C_{D}$ for a circular cylinder are from 1 to 1.2 , we will estimate $C_{D}=1.1$. Using the above values, and an air density $\rho$ of 0.0011 $\mathrm{gm} \mathrm{cm}^{-3}$, we obtain from (13) $\alpha=0.05$. Therefore, a distance of about $40 \mathrm{~cm}$ beyond the velocity jump is required to reduce the speed of such a column to $30 \%$ of the free-stream speed.

The determination of the retardation coefficient $\alpha$ for stellar crystals is difficult because the magnitude of the drag coefficient is not known in this case. However, if the stellar crystal is considered to be composed of three columns which cross each other at their center points to give angles of $60^{\circ}$ between each of the six branches, the area of the stellar will be about three times the area of one isolated column of the same maximum dimension, but the mass $m$ of the stellar will be similar to that of this same isolated column since columns are about three times thicker than stellars (Auer and Veal, 1970). Moreover, the drag coefficient $C_{D}$ for a stellar will be greater than that for an individual column. It follows from these facts that the coefficient $\alpha$ for a stellar crystal will be more than three times that for a columnar ice crystal of the same maximum dimension. Therefore, stellar crystals should undergo substantial retardation in both decelerators.

Using these coefficients and the retardation curve of Fig. 7 along with the measured velocities in the two decelerators given in Figs. 3 and 6, one may predict the behavior in the decelerator of the three crystals considered above. 


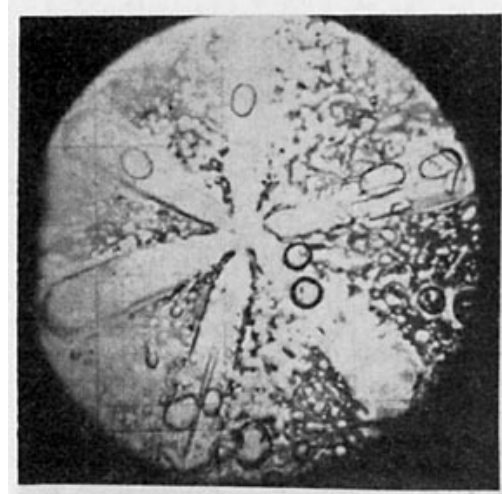

(a)

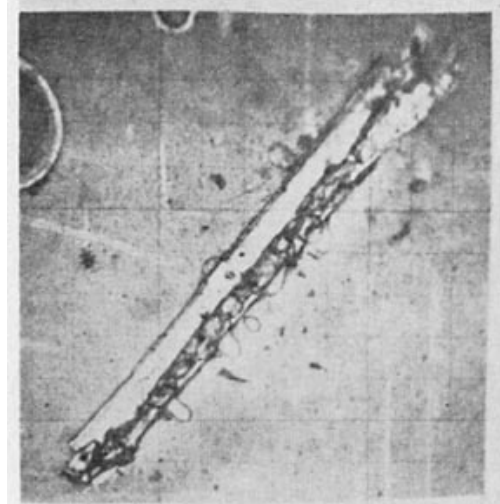

(c)

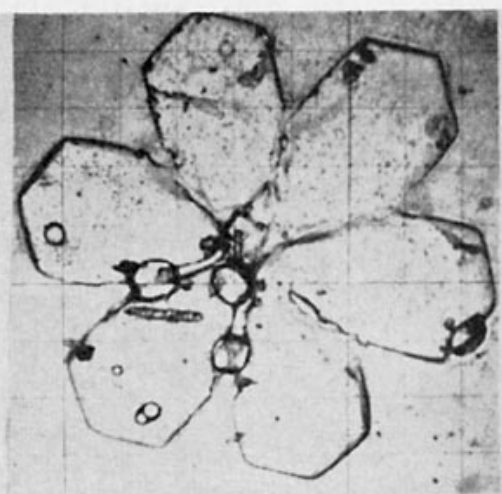

(b)

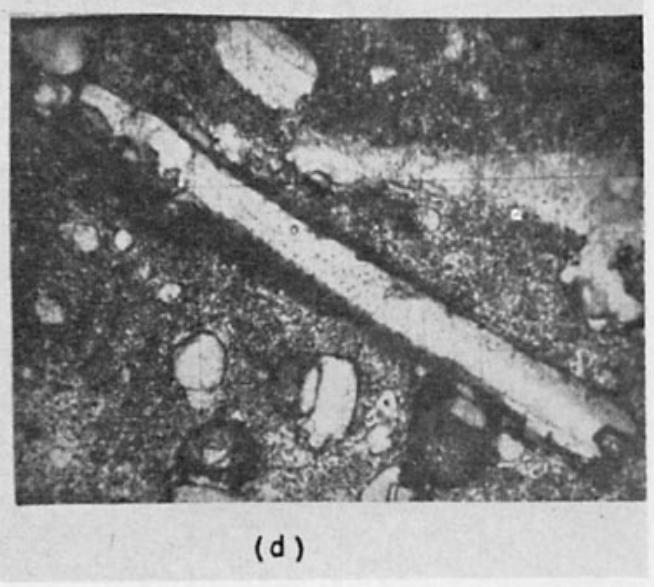

Fig. 8. Four examples of large delicate ice crystals collected intact from aircraft by the use of a decelerator: (a) stellar crystal $1.7 \mathrm{~mm}$ in diameter; (b) crystal with sector-like branches $0.84 \mathrm{~mm}$ in diameter; (c) needle $1.4 \mathrm{~mm}$ long; (d) elementary sheaths $1.1 \mathrm{~mm}$ long. The length of the sides of the square grids in each photograph is $100 \mu \mathrm{m}$.

In decelerator 1 , the velocity jump is seen to occur about $17 \mathrm{~cm}$ ahead of the point at which the particles are collected. Table 1 gives the products $x \alpha$ for the three crystal types and the velocity ratios expected for the crystals at the point of collection. Corresponding values are shown in Table 1 for decelerator 2 based on a velocity jump $53 \mathrm{~cm}$ ahead of the point at which the particles are collected. It can be seen from these results that in decelerator 2 plates, columns and stellars with maximum dimensions of $1 \mathrm{~mm}$ all approach quite closely the full deceleration ratio $(0.2)$ of the airstream. In decelerator 1 the plates and stellars undergo nearly full deceleration but columns $1 \mathrm{~mm}$ in length are only decelerated by a factor of 0.45 at the point of collection.

\section{Results}

The decelerators described above have been used extensively during the past three years. Decelerator 1 is used on light, slow-flying aircraft (e.g., a Cessna 180) and decelerator 2 , which is much longer than decelerator 1 , is used on oụr ressearch aircraft (a B-23) which cruises around $100 \mathrm{~m} \mathrm{sec}-1$. These studies have shown that large, delicate ice crystals, which would normally shatter on collection, can often be collected and replicated without fragmentation when the decelerators are used.

Some examples of the delicate ice crystals which have been collected intact using the decelerators are shown in Fig. 8. Fig. 8a shows a lightly rimed stellar crystal $1.7 \mathrm{~mm}$ in diameter which was collected from an aircraft flying at $30 \mathrm{~m} \mathrm{sec}^{-1}$. Even though this type of crystal is particularly prone to breakage, especially where the arms join at the center, this large crystal was collected intact after being decelerated. A crystal with sectorlike branches $0.84 \mathrm{~mm}$ in diameter is shown in Fig. 8b. This was collected at an aircraft speed of $35 \mathrm{~m}$ $\mathrm{sec}^{-1}$. Fig. $8 \mathrm{c}$ shows a rimed needle $1.4 \mathrm{~mm}$ long which was collected at an aircraft speed of $45 \mathrm{~m} \mathrm{sec}^{-1}$. An elementary sheath $1.1 \mathrm{~mm}$ long and $0.1 \mathrm{~mm}$ wide with a notch at one end is shown in Fig. 8d. This crystal was collected at an aircraft speed of $50 \mathrm{~m} \mathrm{sec}^{-1}$. These examples suffice to demonstrate that with the 
TABLE 2. Empirically deduced estimates of maximum impact speeds which natural ice crystals can withstand without fragmentation when they collide with a Mylar film coated with a wet $4 \%$ solution of Formvar in chloroform $150 \mu \mathrm{m}$ thick.

\begin{tabular}{|c|c|c|}
\hline Type of crystal $\dagger$ & $\begin{array}{c}\text { Maximum } \\
\text { dimensions } \\
\text { of crystal } \\
(\mu \mathrm{m})\end{array}$ & $\begin{array}{c}\text { Maximum impact } \\
\text { speed at } \\
\text { which crystal } \\
\text { remains intact } \\
\left(\mathrm{m} \mathrm{sec}^{-1}\right)\end{array}$ \\
\hline $\begin{array}{l}\text { Stellar and dendritic crystals } \\
\text { (P1b, P1c, Pld, Ple, P1f) }\end{array}$ & 3000 & 9 \\
\hline Thin hexagonal plates (P1a) & 150 & 60 \\
\hline Thin hexagonal plates (Pla) & 1000 & 14 \\
\hline Thick plates (C1g, C1h) & 1500 & 70 \\
\hline Solid columns (C1e, N1e) & 3000 & 27 \\
\hline Hollow columns (C1f) & 3000 & 18 \\
\hline Needles (N1a, N1b, N2a) & 3000 & 9 \\
\hline
\end{tabular}

$\dagger$ Based on classification by Magono and Lee (1966).

use of the decelerators described in this paper much larger ice crystals can be collected from aircraft without the crystals breaking up than is normally possible.

From an analysis of the large number of different types of crystals which have been collected and replicated on a Formvar-coated Mylar film after passing through a decelerator, we have been able to deduce approximate values for the maximum impact velocities which various types and sizes of ice crystals can withstand without fragmentation. These values are listed in Table 2.

A detailed account of the ice crystals collected in different clouds over the Pacific Northwest using the decelerators described in this paper has been given by Hobbs et al. (1971).
Acknowledgments. Thanks are due to Miss J. Fussell for her help in data reduction. This research was supported by the Division of Atmospheric Water Resources Management of the U. S. Department of Interior, Bureau of Reclamation, through a contract with the State of Washington, Department of Ecology, and by the RANN program of the National Science Foundation under NSF Grants GA-17381 and GA-27637.

\section{REFERENCES}

Auer, A. H., Jr., and D. L. Veal, 1970: The dimensions of ice crystals in natural clouds. $J$. Atmos. Sci., 27, 919-926.

Bradshaw, P., and R. C. Pankhurst, 1964: The design of lowspeed wind tunnels. Prog. Aeronaut. Sci., 5, 1-69.

Goldstein, S., Ed., 1938: Modern Developments in Fluid Dynamics. Oxford, Clarendon Press, 330 pp.

Hobbs, P. V., L. F. Radke, A. B. Fraser, J. D. Locatelli, C. E. Robertson, D. G. Atkinson, R. J. Farber, R. R. Weiss and R. C. Easter, 1971: Studies of winter cyclonic storms over the Cascade Mountains (1970-71). Contributions from the Cloud Physics Group, University of Washington, Res. Rept. VI, $306 \mathrm{pp}$.

Lamb, H., 1945: Hydrodynamics. London, Dover, 738 pp.

MacCready, P. B., Jr., and C. J. Todd, 1964: Continuous particle sampler. J. A ppl. Meteor., 3, 450-460.

Magono, C., and C. W. Lee, 1966: Meteorological classification of natural snow crystals. J. Fac. Sci., Hokkaido Univ., Ser. $V I I, 2,321-335$.

Mossop, S. C., and A. Ono, 1969: Measurements of ice crystal concentration in clouds. J. Rech. Atmos., 26, 130-137.

,-- and K. J. Hefferman, 1967: Studies of ice crystals in natural clouds. J. Rech. Atmos., 3, 45-64.

Nakaya, U., 1954: Snow Crystals: Natural and Arificial. Harvard University Press, C., $510 \mathrm{pp}$.

Schlichting, H., 1955: Boundary Layer Theory. New York, McGraw-Hill, $535 \mathrm{pp}$.

Yamashita, A., 1969: Methods of continuous particle sampling by aircraft. J. Meleor. Soc., Japan., 47, 86-97. 